\title{
Minimally invasive treatment of a bulging appendiceal orifice
}

\author{
C. Jorissen ${ }^{1,2}$, F. Lansink ${ }^{1}$, P.J. Cuyle ${ }^{1,2}$, M. Bronswijk $k^{1,2}$ \\ (1) Department of Gastroenterology and Hepatology, Imelda General Hospital, Bonheiden, Belgium; (2) Department of Gastroenterology and Hepatology, University \\ Hospitals Leuven, Leuven, Belgium
}

\section{Question}

A 60-year-old fecal occult blood test-positive patient was referred to our center to undergo colonoscopy. Two diminutive sessile polyps were detected, macroscopically compatible with sessile serrated lesions (SSL), which were resected using cold-snare polypectomy. Furthermore, a bulging appendiceal orifice was identified with mucoid appearance (Figure 1, left). Biopsies were taken and abdominal computed tomography (CT) was performed (Figure 1, right).

What is the diagnosis and which treatment would you consider?

\section{Answer}

Abdominal CT-scan showed a normal appendiceal region, without signs of an underlying mucocele or mass. Surprisingly, histopathological analysis revealed a diagnosis of a large SSL without dysplasia, completely involving the appendiceal orifice.

In the past, $>50 \%$ involvement of the appendiceal orifice has been suggested as a reason to refer patients for surgical resection ${ }^{[1]}$. Since recent years, endoscopic full-thickness resection (eFTR) has broadened the therapeutic horizon of periappendiceal lesions, by providing ease-of-use in complex situations as well as broad resection margins. Although eFTR may avert the need for surgery, a non-negligible risk of appendicitis should be taken into account in this specific context, occurring in up to $14 \%$ of patients ${ }^{[2]}$. In our patient, as ESD and EMR were deemed unfeasible due to complete invasion of the appendiceal orifice, eFTR was performed (Figure 2). Final histopathological analysis revealed a non-dysplastic large SSL with wide clear margins. No complications occurred, after which the patient was discharged the following morning.

While diagnosis of a mucocele should be considered in patients with a bulging peri-appendicular region, our case illustrates that invasion by SSLs can lead to a similar presentation and that endoscopic resection can be considered, even in situations with complete involvement of the appendiceal orifice.

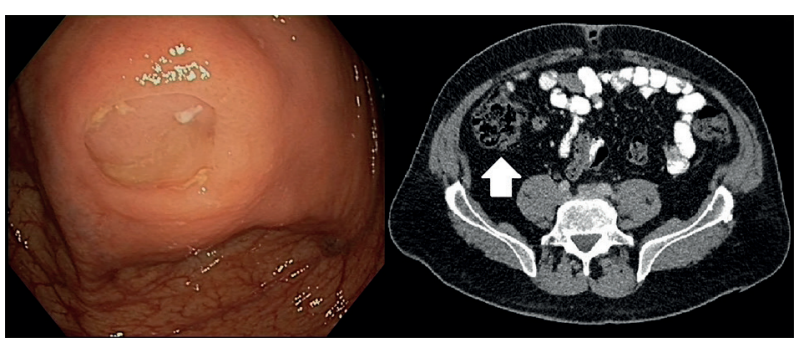

Figure 1

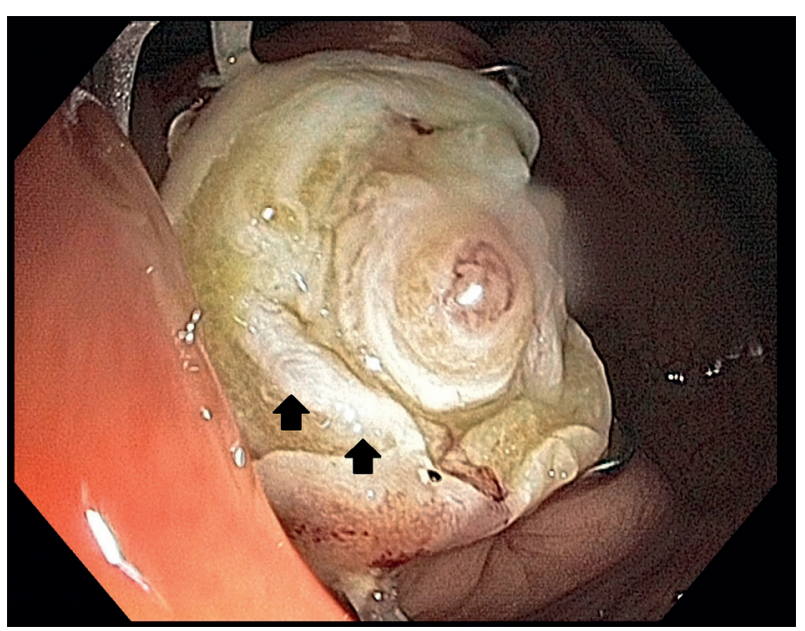

Figure 2

\section{References}

1. TATE DJ, DESOMER L, AWADIE H, GOODRICK K, HOURIGAN L, SINGH R, et al. EMR of laterally spreading lesions around or involving the appendiceal orifice: technique, risk factors for failure, and outcomes of a tertiary referral cohort. Gastrointest Endosc. 2018, 87:1279-1288.e2.

2. SCHMIDBAUR S, WANNHOFF A, WALTER B, MEIER B, SCHAFER C, MEINING A, et al. Risk of appendicitis after endoscopic full-thickness resection of lesions involving the appendiceal orifice: a retrospective analysis. Endoscopy. 2020, Epub ahead of print.

Correspondence to: Michiel Bronswijk, Imeldalaan 9, 2820, Bonheiden, Belgium. Email: mjh.bronswijk@gmail.com

Submission date : 23/10/2020

Acceptance date : 02/12/2020 